\title{
Effect of a twin-emitter design strategy on a previously reported thermally activated delayed fluorescence organic light-emitting diode
}

\author{
Ettore Crovini ${ }^{\ddagger 1}$, Zhen Zhang ${ }^{\ddagger 2}$, Yu Kusakabe ${ }^{3}$, Yongxia Ren ${ }^{3}$, Yoshimasa Wada ${ }^{3}$, \\ Bilal A. Naqvi ${ }^{4}$, Prakhar Sahay ${ }^{4}$, Tomas Matulaitis ${ }^{1}$, Stefan Diesing ${ }^{1,5}$, \\ Ifor D. W. Samuel ${ }^{5}$, Wolfgang Brütting ${ }^{4}$, Katsuaki Suzuki ${ }^{3}$, Hironori Kaji ${ }^{* 3}$, \\ Stefan Bräse ${ }^{\star 2,6, \S}$ and Eli Zysman-Colman ${ }^{*} 1$, 凡
}

\section{Full Research Paper}

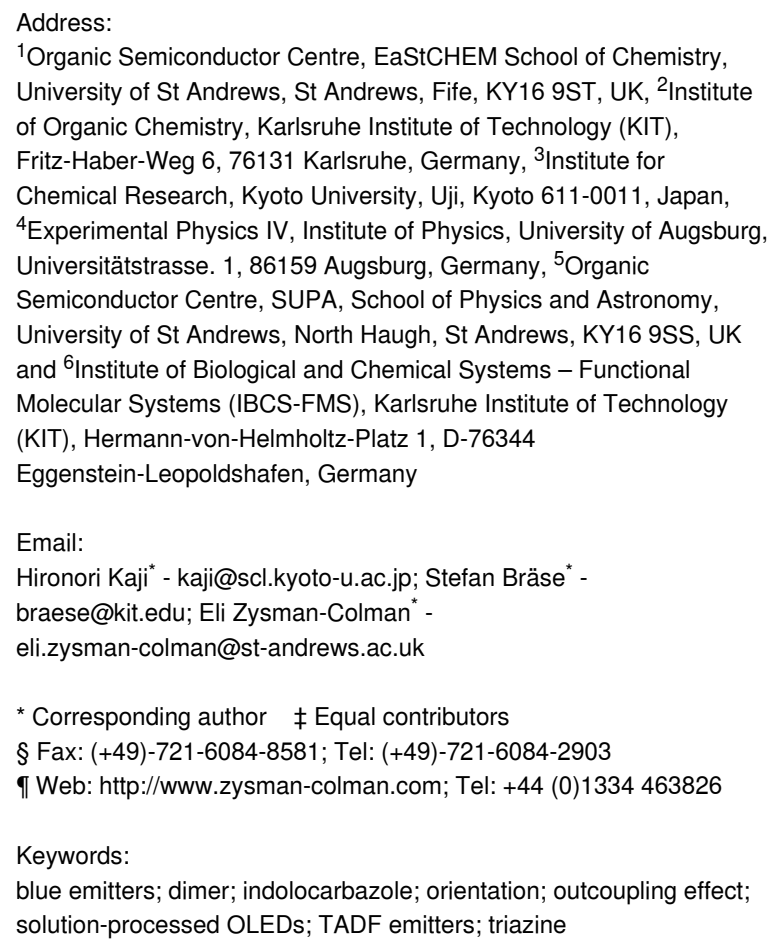

${ }^{1}$ Organic Semiconductor Centre, EaStCHEM School of Chemistry, University of St Andrews, St Andrews, Fife, KY16 9ST, UK, ${ }^{2}$ Institute of Organic Chemistry, Karlsruhe Institute of Technology (KIT), Fritz-Haber-Weg 6, 76131 Karlsruhe, Germany, ${ }^{3}$ Institute for Chemical Research, Kyoto University, Uji, Kyoto 611-0011, Japan, ${ }^{4}$ Experimental Physics IV, Institute of Physics, University of Augsburg, Universitätstrasse. 1, 86159 Augsburg, Germany, ${ }^{5}$ Organic Semiconductor Centre, SUPA, School of Physics and Astronomy, University of St Andrews, North Haugh, St Andrews, KY16 9SS, UK and ${ }^{6}$ Institute of Biological and Chemical Systems - Functional Molecular Systems (IBCS-FMS), Karlsruhe Institute of Technology (KIT), Hermann-von-Helmholtz-Platz 1, D-76344 Eggenstein-Leopoldshafen, Germany

Email:

Hironori Kaji - kaji@scl.kyoto-u.ac.jp; Stefan Bräse* braese@kit.edu; Eli Zysman-Colman* eli.zysman-colman@st-andrews.ac.uk

* Corresponding author $\ddagger$ Equal contributors § Fax: (+49)-721-6084-8581; Tel: (+49)-721-6084-2903 I Web: http://www.zysman-colman.com; Tel: +44 (0)1334 463826

Keywords:

blue emitters; dimer; indolocarbazole; orientation; outcoupling effect; solution-processed OLEDs; TADF emitters; triazine

\author{
Beilstein J. Org. Chem. 2021, 17, 2894-2905. \\ https://doi.org/10.3762/bjoc.17.197 \\ Received: 02 September 2021 \\ Accepted: 19 November 2021 \\ Published: 08 December 2021 \\ Associate Editor: P. J. Skabara \\ (C) 2021 Crovini et al.; licensee Beilstein-Institut. \\ License and terms: see end of document.
}

\begin{abstract}
In this work we showcase the emitter DICzTRZ in which we employed a twin-emitter design of our previously reported material, ICzTRZ. This new system presented a red-shifted emission at $488 \mathrm{~nm}$ compared to that of ICzTRZ at $475 \mathrm{~nm}$ and showed a comparable photoluminescence quantum yield of $57.1 \%$ in a $20 \mathrm{wt} \% \mathrm{CzSi}$ film versus $63.3 \%$ for ICzTRZ. The emitter was then incorporated within a solution-processed organic light-emitting diode that showed a maximum external quantum efficiency of $8.4 \%$, with Commission Internationale de l'Éclairage coordinate of $(0.22,0.47)$, at $1 \mathrm{~mA} \mathrm{~cm}^{-2}$.
\end{abstract}




\section{Introduction}

Organic thermally activated delayed fluorescence (TADF) materials have elicited tremendous excitement as an alternative to phosphorescent complexes in organic light-emitting diodes (OLEDs) because these organic compounds can also achieve a theoretical 100\% internal quantum efficiency (IQE) but do not require the use of scarce, noble metals $[1,2]$. Since the luminescence in an OLED is achieved through the radiative decay of electrically generated excitons, high-efficiency devices must be able to harvest both the $25 \%$ singlet and $75 \%$ triplet excitons to produce light [3]. Distinct from phosphorescent compounds, TADF molecules harvest triplet excitons by converting them into emissive singlets via a reverse intersystem crossing (RISC) mechanism. This mechanism is operational when the energy gap $\left(\Delta E_{\mathrm{ST}}\right)$ between the lowest-lying singlet and triplet excited states $\left(\mathrm{S}_{1}\right.$ and $\left.\mathrm{T}_{1}\right)$ is sufficiently small and spin-orbit coupling (SOC) is non-negligible [4-7]. This small $\Delta E_{\mathrm{ST}}$ can be achieved by spatially separating the highest occupied molecular orbital (HOMO) and the lowest unoccupied molecular orbital (LUMO), thereby reducing the exchange integral of these two orbitals determining the energies of the $S_{1}$ and $T_{1}$ states relative to the ground state. The spatial separation of the HOMO and LUMO on donor and acceptor, respectively, will result in an $\mathrm{S}_{1} \rightarrow \mathrm{S}_{0}$ transition with predominantly charge transfer (CT) character. Highly twisted donor-acceptor architectures are typically employed to realize small $\Delta E_{\mathrm{ST}}[4,8]$. SOC can be enhanced by ensuring that the nature of the $S_{1}$ and $T_{1}$ states is different, for example by additionally involving a third (local) triplet state with different symmetry, because otherwise SOC vanishes when the orbital types for these two states are the same, according to El-Sayed's rule [9].

Designing a molecule able to achieve RISC and the desired $100 \%$ IQE is just the first step toward an efficient OLED since the light needs to escape the device. A device is composed of a stack of several layers of organic semiconductor materials, each possessing different refractive indices, sandwiched between two electrodes. Depending on the angle of emission of the light with respect to the plane of the device, total internal reflection at the organic-glass as well as the glass-air interfaces can occur as can coupling to surface plasmon polaritons (SPP) at the interface with the cathode, all contributing to decreasing the external quantum efficiency (EQE) of the device. A compound will emit light perpendicular to its transition dipole moment (TDM), quantified by the anisotropy factor, $a$. Controlling the orientation of the TDM to lie horizontally in the film (where $1-a$ is the fraction of horizontally aligned TDMs) will lead to a maximized amount of light exiting the device. However, when the transition dipoles of the emitter are randomly oriented then only around $20 \%$ of the light can escape the device [10].
Indolocarbazole ( $\mathbf{I C z}$ )-based emitters have been recently employed in several high-performance and highly horizontally oriented materials. ICz acts as a weak, planar, and rigid donor [11-14]. Examples of compounds incorporating an $\mathrm{ICz}$ unit include reports from Xiang et al. with the emitters IndCzpTr-1 and IndCzpTr-2 [11], and Maeng et al. with the emitter TRZTPDICz [12] (see Figure 1). In the doped film, IndCzpTr-1 and IndCzpTr-2 present high photoluminescence quantum yields, $\Phi_{\mathrm{PL}}$, of $75.2 \%$ and $71.9 \%$, respectively, and delayed fluorescence lifetimes, $\tau_{\mathrm{d}}$, of $25.48 \mu \mathrm{s}$ and $34.31 \mu \mathrm{s}$, respective1y. The devices produced with these materials reached maximum external quantum efficiencies $\left(\mathrm{EQE}_{\max }\right)$ values of $14.5 \%$ and $30 \%$ at low brightness, but efficiency roll-off was significant, with $\mathrm{EQE}$ at $100 \mathrm{~cd} \mathrm{~m}^{-2}, \mathrm{EQE}_{100}$, of $11.0 \%$ and $15.3 \%$ for the OLEDs with IndCzpTr-1 and IndCzpTr-2, respectively. The addition of two phenyl units on the ICz in TRZTPDICz increased the donor strength and led to $\Phi_{\mathrm{PL}}$ of near unity $(96 \%)$ and a much shorter $\tau_{\mathrm{d}}$ of $8.57 \mu \mathrm{s}$ in $20 \mathrm{wt} \%$ DBFPO film (DBFPO $=2,8$-bis (diphenylphosphine oxide)dibenzofuran). The device made from this material has a very high $\mathrm{EQE}_{\max }$ of $30.3 \%$, which decreases to $18.4 \%$ at $1000 \mathrm{~cd} \mathrm{~m}^{-2}$; the use of a stronger donor in TRZ-TPDICz results in a red-shift of the electroluminescence, compared to IndCzpTr-1 and IndCzpTr-2 (the electroluminescence maximum wavelength, $\lambda_{\mathrm{EL}}$ of $472 \mathrm{~nm}$ and $496 \mathrm{~nm}$ for IndCzpTr-1 and IndCzpTr-2, respectively, against $\lambda_{\mathrm{EL}}$ of $509 \mathrm{~nm}$ for TRZ-TPDICz). In our previous work, we presented the first example of a di-functionalized ICz-based emitter ICzTRZ [13,14], that presented nearly complete horizontal orientation in a wide number of host matrices. The best combination of properties was obtained in $\mathrm{mCBP}$ as a host, with the photoluminescence maximum wavelength, $\lambda_{\mathrm{PL}}$ of $479 \mathrm{~nm}, \Phi_{\mathrm{PL}}$ of $70 \%$, and a $\tau_{\mathrm{d}}$ of $121.1 \mu$ s for the vacuumdeposited doped film. The anisotropy factor $(a)$ in $5 \mathrm{wt} \%$ mCBP film is 0.09 , indicating a very high degree of horizontal orientation (91\%), which together with the high $\Phi_{\mathrm{PL}}$ led to a high-performing device with $\mathrm{EQE}_{\max }$ of $22.1 \%$ (Figure 1).

It has been documented in the literature that some multichromophore emitters show enhanced molar extinction coefficients of absorption and high $\Phi_{\mathrm{PL}}$ [15-18]. This led to OLEDs employing dual or multi emitter-designed compounds to show much improved $\mathrm{EQE}_{\max }$ compared to devices with their singleemitter counterparts (Figure 1), albeit with a red-shifted emission [16-19]. The advantages of the dual-emitter design are best illustrated by the cross-comparison of CzTRZ [20,21], a molecule that did not present any TADF and thus the OLED showed a low $\mathrm{EQE}_{\max }$ of $5.8 \%$, while the emitter, 33TCzTTrz [22], is TADF and the OLED showed a much superior $\mathrm{EQE}_{\max }$ of $25.0 \%$. There is a significant red-shift of the electrolumines- 


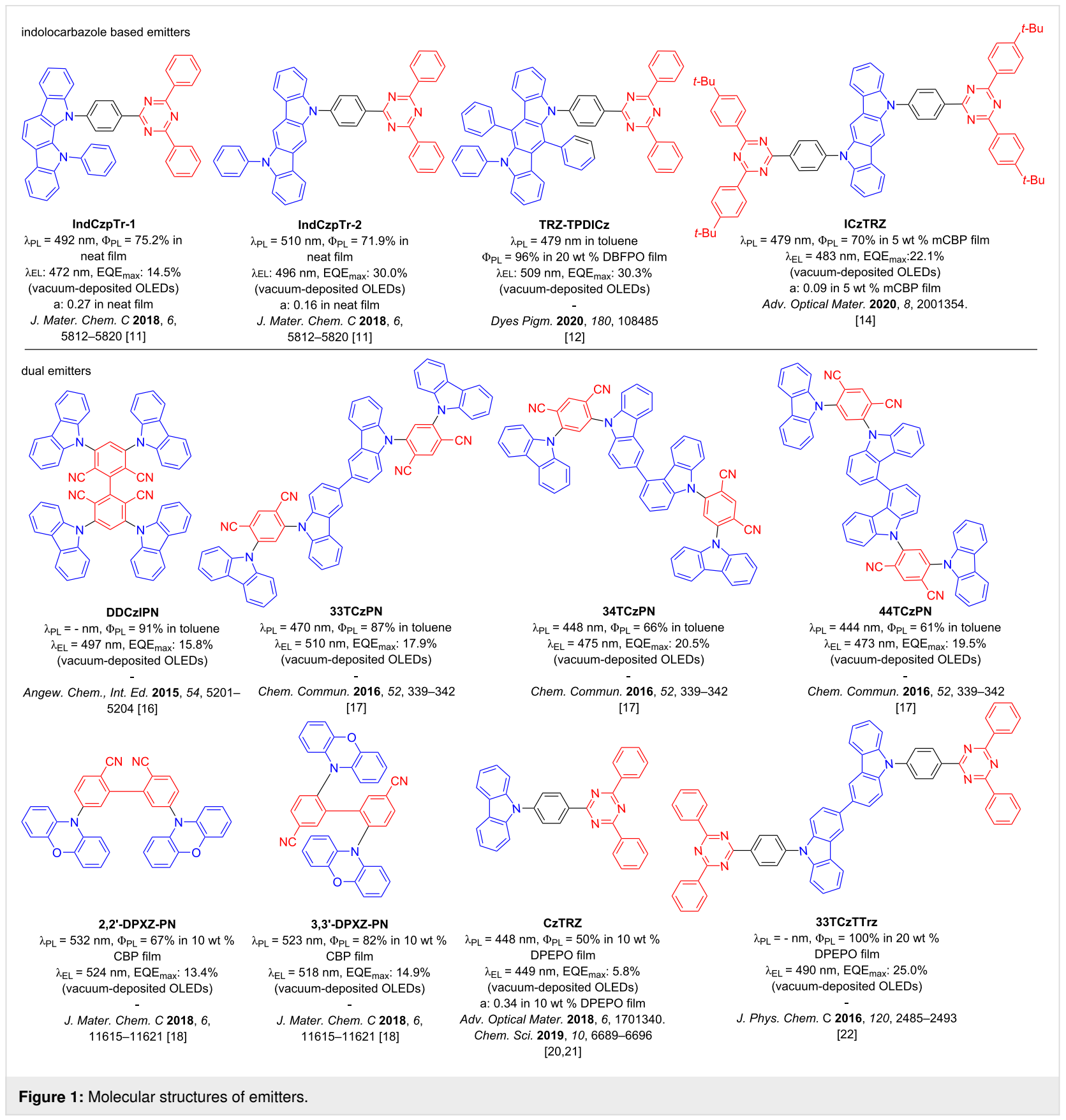

cence, with $\lambda_{\text {EL }}$ going from $449 \mathrm{~nm}$ for CzTRZ to $490 \mathrm{~nm}$ for

\section{TCzTTrz.}

In this work, we utilized a similar strategy to assess the change in optoelectronic properties and device performance of the compound DICzTRZ (Figure 2) compared to our recently reported ICzTRZ study [14]. We note that the effective doubling of the molecular weight necessitates that we fabricate solutionprocessed devices. Importantly, solution-processed films tend to present isotropic orientation [10] due to the slower deposition times coupled with higher degree of freedom of movement in the solution, unlike the orientation of the emitter in vacuumdeposited films, which occurs only at the surface of the film where the emitter orientation is then "frozen" into place once additional layers of material have covered it. While this loss of controlled orientation in the solution-processed film is true for small molecules, polymers and other high molecular weight emitters can show at least some degree of orientation in solution-processed films. For instance, Senes et al. [23,24]. showed that the OPVn series of polymers exhibited higher horizontal orientation by increasing the length of the polymer chain, and by extension the molecule. Considering the high degree of hori- 


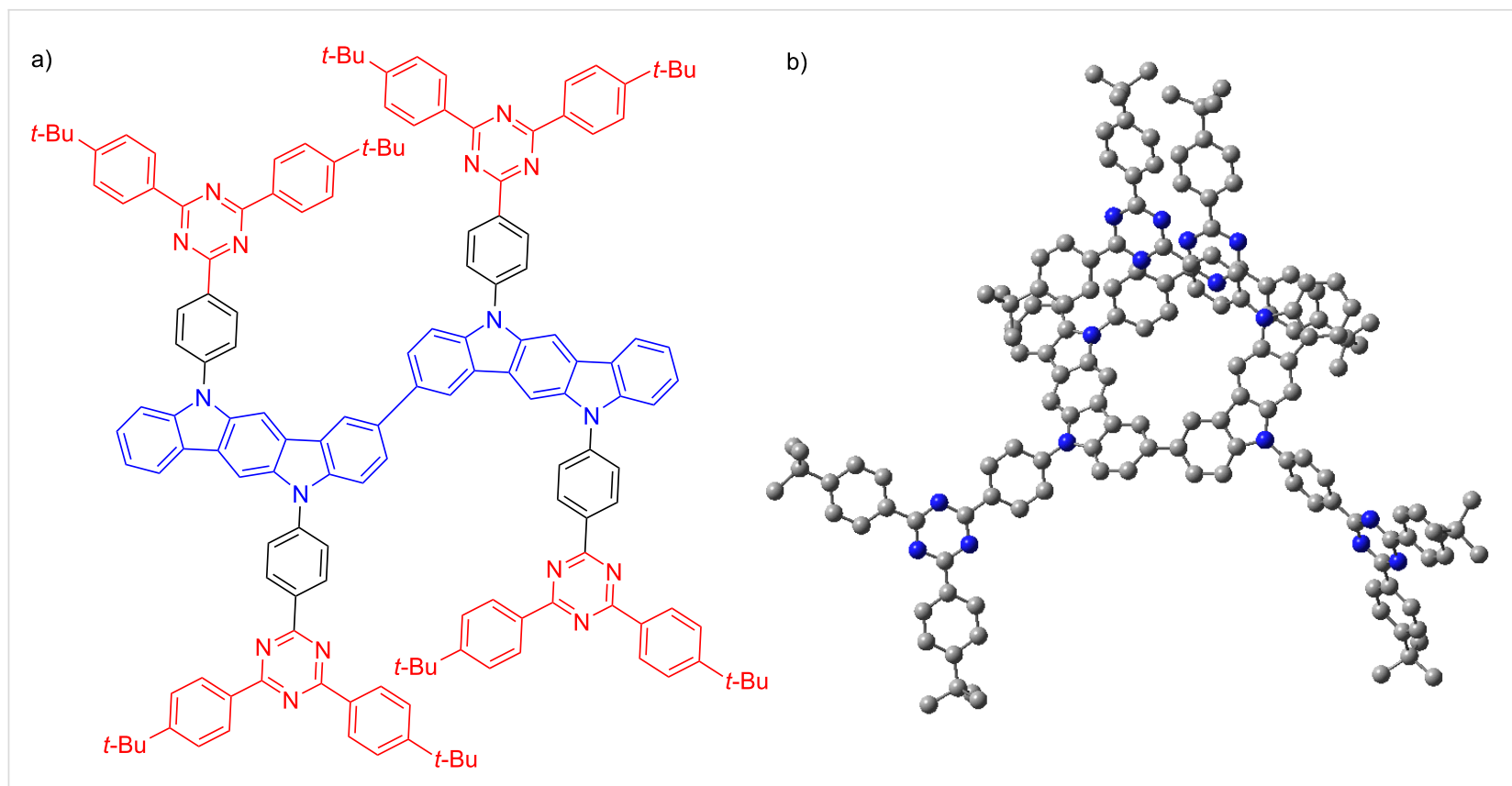

Figure 2: a) Molecular structure and b) optimized DFT-calculated geometry of DICzTRZ. Hydrogen atoms are omitted for clarity.

zontal orientation that ICzTRZ already showed in vacuumdeposited films (anisotropy factor of 0.09 in $10 \mathrm{wt} \%$ film of $\mathrm{mCBP}$ ) and the high molecular weight of DICzTRZ, we hypothesized that DICzTRZ may also present horizontal orientation in the film and subsequently improve light outcoupling in the device.

\section{Results and Discussion \\ Synthesis}

The oxidative coupling conditions for the synthesis of carbazole dimers were initially applied to access the dimer of ICzTRZ $[25,26]$. Treating ICzTRZ with $\mathrm{FeCl}_{3}$ in dichloromethane (DCM) at room temperature for 12 hours did not lead to any product formation. However, when the temperature was increased to $40{ }^{\circ} \mathrm{C}$, DICzTRZ was formed and was isolated in a yield of $20 \%$, while increasing the temperature to $60{ }^{\circ} \mathrm{C}$ resulted in complete consumption of the starting material and DICzTRZ was isolated in $66 \%$ yield. The identity and purity of DICzTRZ were determined by a combination of NMR spectroscopy, mass spectrometry, and IR spectroscopy.

\section{Theoretical calculations}

Density functional theory (DFT) and time-dependent DFT (TDDFT) calculations in the gas phase at the PBE0/6-31G(d,p) level reveal the potential of DICzTRZ as a TADF material. The nature of the $S_{1}$ and $T_{1}$ states and their corresponding energies were then obtained using the Tamm-Dancoff approximation [27] to TD-DFT (TDA-DFT). DICzTRZ possesses a $\Delta E_{\mathrm{ST}}$ of $0.19 \mathrm{eV}$, comparable to $0.22 \mathrm{eV}$ obtained for ICzTRZ at the same level of theory. We can observe a slightly stabilized $\mathrm{S}_{1}$ energy of $2.83 \mathrm{eV}$ (2.92 eV for ICzTRZ) and $\mathrm{T}_{1}$ energy of $2.64 \mathrm{eV}$ (2.70 eV for ICzTRZ) [14] compared to those of ICzTRZ. Compared to ICzTRZ, there is a much higher density of intermediate triplet states between $S_{1}$ and $T_{1}$, which is expected to enhance the efficiency of the RISC process due to the presence of increased spin-vibronic coupling [28-33]. The permanent dipole moment (PDM) of DICzTRZ is substantial increased to 2.1 Debye compared to that in ICzTRZ (0.3 Debye); however, both the transition dipole moment (TDM) and oscillator strength $(f)$ are slightly smaller (TDM = 7.6 Debye and $f=0.62$ ) than the values calculated for ICzTRZ (TDM $=7.9$ Debye and $f=0.72$ ). DICzTRZ shows a shallower $\mathrm{HOMO}$ at $-5.03 \mathrm{eV}$, reflective of a certain degree of conjugation between the two indolocarbazole moieties, compared to the HOMO of ICzTRZ $(-5.19 \mathrm{eV})$. The LUMO level remains essentially unchanged $(-1.76 \mathrm{eV}$ for DICzTRZ vs $-1.75 \mathrm{eV}$ for ICzTRZ) since the electronic environment surrounding the $t$-Bu-triazine remains essentially unperturbed (Figure 3 ).

\section{Optoelectronic properties}

The electrochemical properties of the two materials were studied in degassed DCM with tetra- $n$-butylammonium hexafluorophosphate as the electrolyte and $\mathrm{Fc} / \mathrm{Fc}^{+}$as the internal reference, data are reported versus a saturated calomel electrode (SCE). In both DICzTRZ and ICzTRZ [14] we observed a reversible oxidation wave with respective oxidation potential $\left(E_{\mathrm{ox}}\right)$ at $0.87 \mathrm{~V}$ and $0.96 \mathrm{~V}$ vs SCE (Figure $4 \mathrm{a}$ ). Both compounds also present a second oxidation wave that is more 

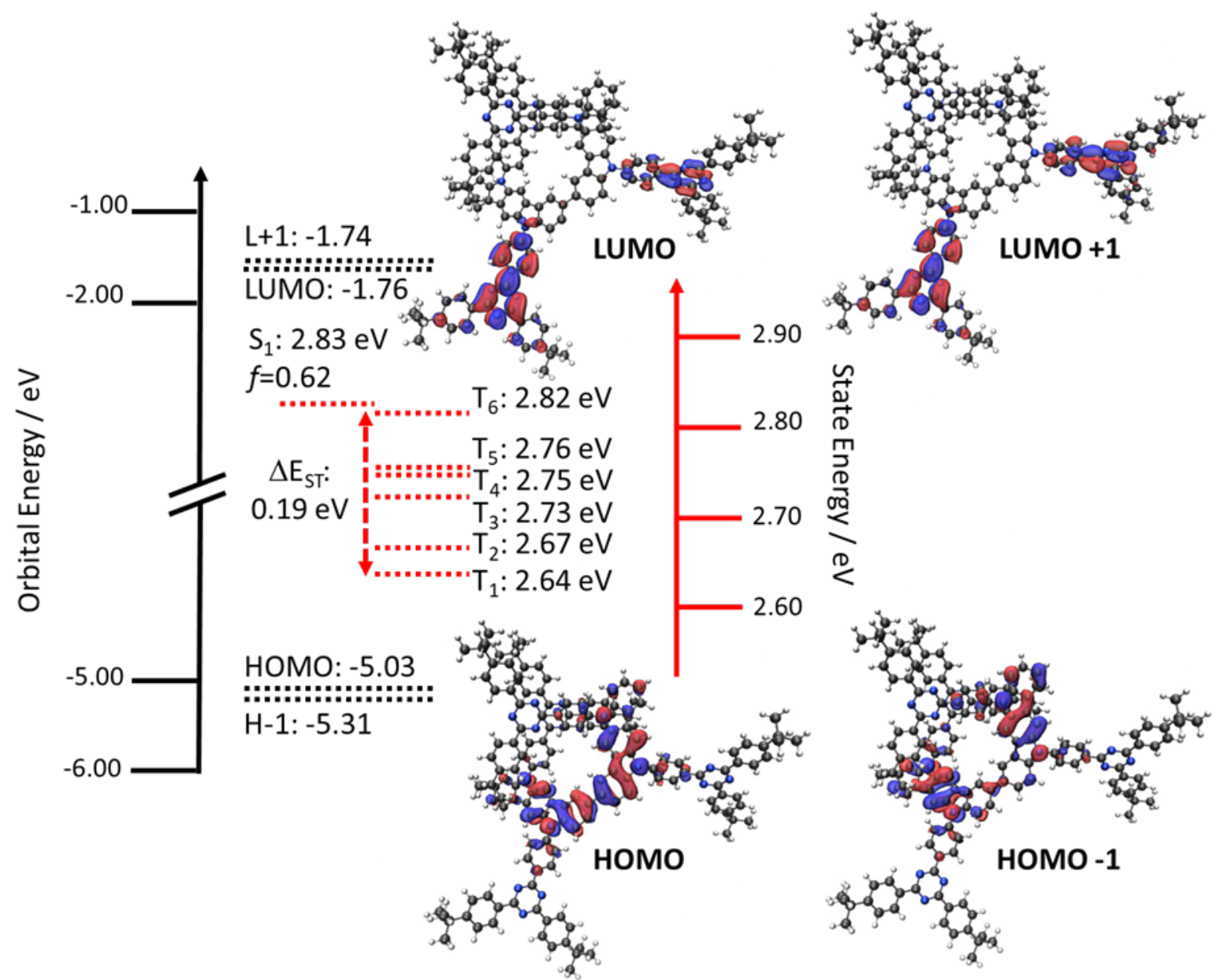

Figure 3: HOMO, HOMO-1 ( $\mathrm{H}-1)$, LUMO, and LUMO+1 $(\mathrm{L}+1)$ electron density distributions (isovalue: 0.02$)$ and energy levels, excited state energy levels.

prominent and cathodically shifted for DICzTRZ at $1.05 \mathrm{~V}$, compared to $1.14 \mathrm{~V}$ for ICzTRZ. No reduction wave is observed for DICzTRZ. The HOMO value calculated from the oxidation potential obtained from differential pulse voltammetry (DPV), is $-5.21 \mathrm{eV}$, which is stabilized compared to that predicted from DFT ( $E_{\mathrm{HOMO}}:-5.03 \mathrm{eV}$ ); however, the less positive oxidation potentials in DICzTRZ versus ICzTRZ does align with the predictions obtained by DFT.

The UV-vis absorption spectrum of DICzTRZ, while slightly red-shifted and with higher molar absorptivity (as was the case for previously published multichromophore materials) [1517,34,35], coincides closely with the one from ICzTRZ [14] (Figure 4b) and also with other indolocarbazole-based com- pounds [11]. The nearly identical profile leads us to conclude that the character of the transitions is likely to be very similar to those associated with ICzTRZ. The two absorption bands located between 330 and $350 \mathrm{~nm}$ are ascribed to the LE transitions within the diindolocarbazole donor. The two lower energy and lower absorptivity bands at $390 \mathrm{~nm}$ and $410 \mathrm{~nm}$ are both assigned to CT-type transitions (Figure $4 \mathrm{~b}$ ).

Solvatochromic studies for DICzTRZ show that the PDM of the ground state structure is small and so the absorption spectrum is essentially not affected by changes in polarity, while the excited state shows the characteristic positive solvatochromism associated with an emission from a CT state $\lambda_{\mathrm{PL}}$ going from $462 \mathrm{~nm}$ in the least polar methylcyclohexane to $548 \mathrm{~nm}$ in the 
a)
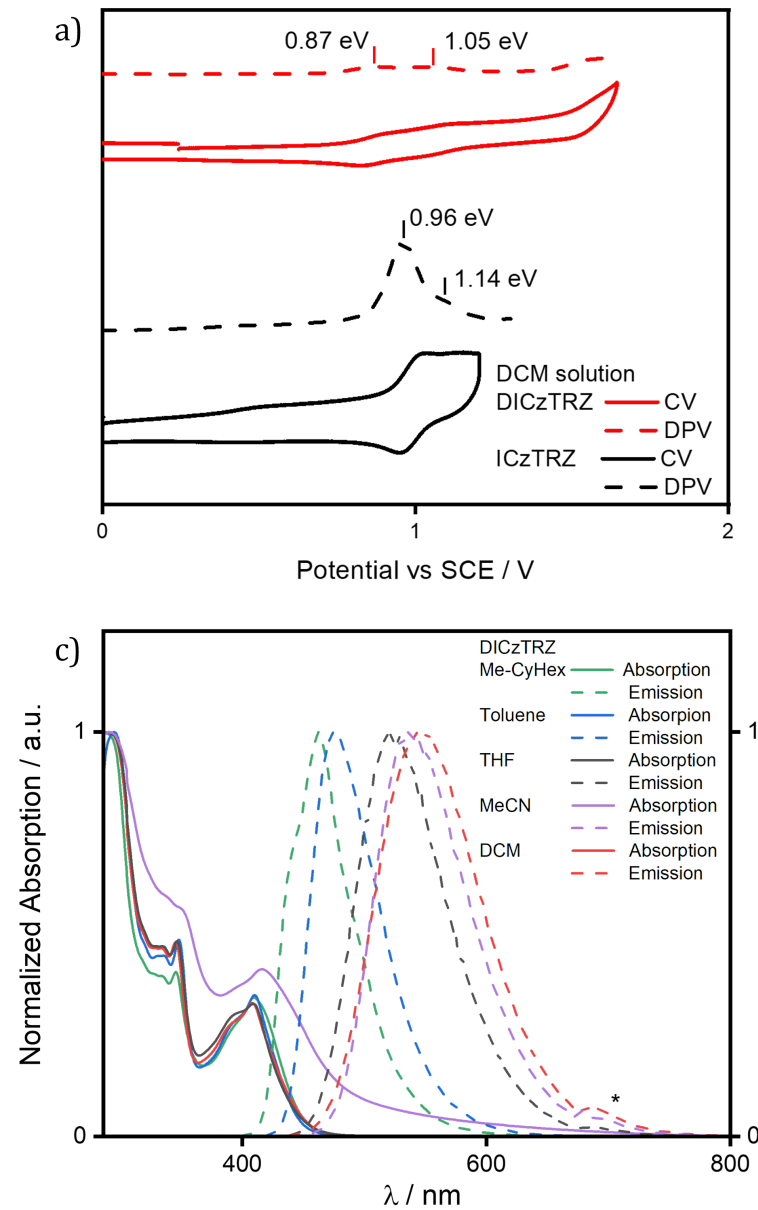

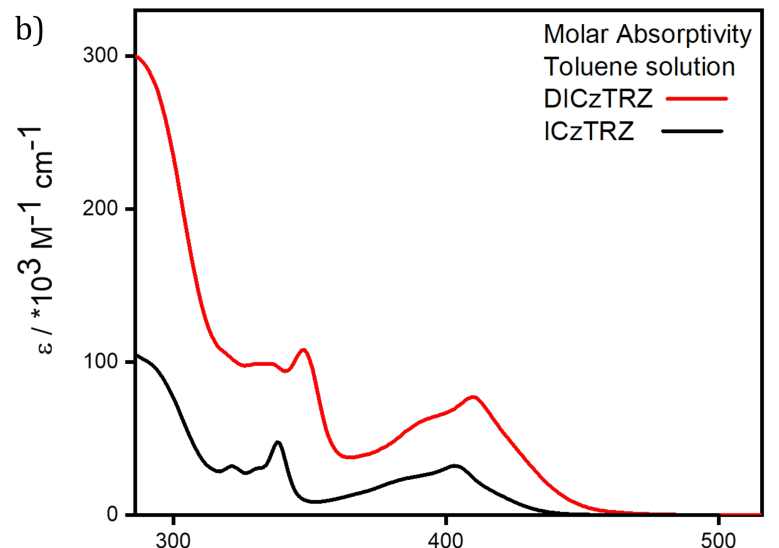

$\lambda / \mathrm{nm}$

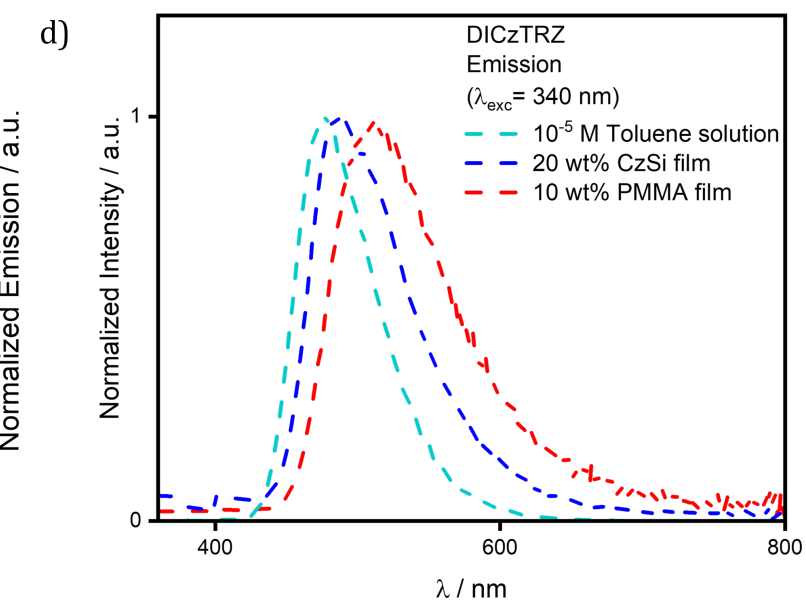

Figure 4: a) Cyclic voltammetry (CV) and differential pulse voltammetry (DPV) of DICzTRZ in DCM (scan rate = $100 \mathrm{mV} / \mathrm{s}$ ). b) UV-vis absorption spectrum of DICzTRZ in $10^{-5} \mathrm{M}$ toluene solution. c) Ground and excited state solvatochromism study of DICzTRZ (excitation wavelength, $\lambda_{\text {exc }}$, = $340 \mathrm{~nm},{ }^{*}=$ second harmonic of the excitation source); d) emission spectra of DICzTRZ in $10^{-5} \mathrm{M}$ toluene solution (cyan), $20 \mathrm{wt} \%$ CzSi film (blue), and $10 \mathrm{wt} \%$ PMMA film (red), $\left(\lambda_{\text {exc }}=340 \mathrm{~nm}\right)$.

most polar dichloromethane). From the previously calculated HOMO level determined from DPV and the optical gap obtained from the intersection of the normalized absorption and emission spectra in DCM $\left(E_{\text {gap }}=2.71 \mathrm{eV}\right)$, we were able to obtain a LUMO energy value of $-2.50 \mathrm{eV}$ (Figure 4c).

The emission of DICzTRZ in degassed toluene is red-shifted at $477 \mathrm{~nm}$ compared to ICzTRZ [14], at $462 \mathrm{~nm}$ (Figure 4d). The excitation spectrum mirrors the profile of the UV-vis absorption (Supporting Information File 1, Figure S4a). Transient PL measurements in degassed toluene show mono-exponential prompt and delayed fluorescence decays at $8.94 \mathrm{~ns}$ and $28.83 \mu \mathrm{s}$, respectively (Supporting Information File 1, Figure $\mathrm{S} 4 \mathrm{c}, \mathrm{d})$. After exposure to oxygen, the delayed fluorescence disappears while the prompt decay lifetime, $\tau_{\mathrm{p}}$, is slightly reduced to $6.80 \mathrm{~ns}$, implying the involvement of triplet states in the emission. When compared to ICzTRZ in degassed toluene,
DICzTRZ presents comparable $\tau_{\mathrm{p}}(9.0 \mathrm{~ns}$ for ICzTRZ), while we observe a substantial one order of magnitude decrease in the delayed lifetime, $\tau_{\mathrm{d}},(229.2 \mu$ s for ICzTRZ [14]), reflective of a more efficient RISC process. DICzTRZ is less emissive than ICzTRZ ( $\Phi_{\mathrm{PL}}$ of $72 \%$ [14]), with $\Phi_{\mathrm{PL}}$ in degassed toluene of $60 \%$ that decreases to $44 \%$ once exposed to oxygen. This reduction in $\Phi_{\mathrm{PL}}$ is in part due to the decrease in the radiative decay rate given the smaller calculated oscillator strength for the emissive $S_{1}$ state for this compound compared to ICzTRZ. The $\Delta E_{\mathrm{ST}}$ of DICzTRZ in toluene glass at $77 \mathrm{~K}$ is $0.21 \mathrm{eV}$ (see Supporting Information File 1, Figure S4, which is significantly smaller than the $0.32 \mathrm{eV}$ obtained for ICzTRZ under the same conditions. The $\mathrm{T}_{1}$ levels of both DICzTRZ and ICzTRZ are comparable at $2.59 \mathrm{eV}$ and $2.62 \mathrm{eV}$, respectively, while the $\mathrm{S}_{1}$ level for DICzTRZ is more stabilized at $2.80 \mathrm{eV}$ vs $2.94 \mathrm{eV}$ for ICzTRZ). We can clearly observe that the phosphorescence spectrum presents a well-defined structure, typical for transi- 
tions coming from a local excited (LE) type state on the diindolocarbazole. TDA-DFT calculations in the gas phase predict that the $\mathrm{T}_{1}$ state is $\mathrm{CT}$ in nature while the lowest-lying triplet states with $L E$ character are $T_{3}$ and $T_{4}\left(T_{3}\right.$ and $T_{4}$ are at $2.73 \mathrm{eV}$ and $2.75 \mathrm{eV}$, respectively, while $\mathrm{T}_{1}$ is at $2.64 \mathrm{eV}$, see Supporting Information File 1, Table S1 and Figure S3). The character of the different transitions was also evaluated by analysis of the natural transition orbitals (NTOs) (see Supporting Information File 1, Table S2). The $\mathrm{T}_{1}$ and $\mathrm{T}_{2}$ HONTO and LUNTO (highest occupied and lowest unoccupied natural Transition orbitals) are localized on the central diindolocarbazole and adjacent triazine, respectively, showing a clear CT between donor and acceptor moieties in the molecule. As previously mentioned, $\mathrm{T}_{3}$ and $\mathrm{T}_{4}$ present $\mathrm{LE}$ character, with the NTOs localized mainly the central di-indolocarbazole. The character of each of $\mathrm{T}_{5}$ and $\mathrm{T}_{6}$ is more difficult to assign as the electron density of the transition is localized on one of the indolocarbazole-triazine fragments and showing a high degree of overlap between the HONTO and LUNTO, which indicates a transition with a mixed $\mathrm{CT}$ and LE character. $\mathrm{S}_{1}$ also presents a clear $\mathrm{CT}$ transition from the diindolocarbazole to the triazine.

With a view to incorporating DICzTRZ as the emitter in a solution-processed OLED, we next investigated the photophysical behavior of this compound in solid host matrices. We began with 10 wt \% doped film of DICzTRZ in PMMA as the polarity of PMMA emulates well that of toluene [36]. The emission maximum in PMMA is $514 \mathrm{~nm}$ (Supporting Information File 1, Figure S5a) with a corresponding $\Phi_{\mathrm{PL}}$ of $29 \%$ under $\mathrm{N}_{2}$. The significantly red-shifted emission in the PMMA film compared to that in toluene coupled with a significantly lower $\Phi_{\mathrm{PL}}$ is suggestive that aggregation-caused quenching is prevalent in this host matrix. Transient PL measurement (Supporting Information File 1, Figure S5b,c) showed multiexponential decay kinetics and lifetimes with an average $\tau_{\mathrm{p}}$ of $8.6 \mathrm{~ns}\left[\tau_{1}=3.5 \mathrm{~ns}\right.$ $\left.(37.5 \%), \tau_{2}=11.6 \mathrm{~ns}(62.5 \%)\right]$ and an average $\tau_{\mathrm{d}}$ of $156.1 \mu \mathrm{s}$ $\left[\tau_{1}=27.98 \mu \mathrm{s}(39.5 \%), \tau_{2}=239.7 \mu \mathrm{s}(60.5 \%)\right]$, respectively. The average prompt fluorescence lifetimes are of a similar magnitude to that of $\operatorname{ICzTRZ}\left(\tau_{\mathrm{p}}=11.5 \mathrm{~ns}\right.$ ) [14] while the average delayed fluorescence decays much faster for DICzTRZ $\left(\tau_{\mathrm{d}}=\right.$ $252.8 \mu$ s for ICzTRZ) [14]. We next focused on the photophysical study in a suitably high triplet energy small molecule host material, CzSi (9-(4-tert-butylphenyl)-3,6-bis(triphenylsilyl)$9 H$-carbazole). The emission in $\mathrm{CzSi}$ at $488 \mathrm{~nm}$, is only slightly red-shifted compared to that in toluene (Figure 4d). Gratifyingly, the $\Phi_{\mathrm{PL}}$ is substantially higher at $57 \%$ in $20 \mathrm{wt} \%$ doped CzSi film, compared to that in the $10 \mathrm{wt} \%$ PMMA films (Table 1). In this host, transient PL measurements show the presence of both prompt and delayed fluorescence (Figure 5a,b) with respective average lifetimes of $\tau_{\mathrm{p}}$ of $7.7 \mathrm{~ns}\left[\tau_{1}=3.8 \mathrm{~ns}\right.$ $\left.(42.5 \%), \tau_{2}=10.6 \mathrm{~ns}(57.6 \%)\right]$ and $\tau_{\mathrm{d}}$ of $69.49 \mu \mathrm{s}$ $\left[\tau_{1}=23.07 \mu \mathrm{s}(49.6 \%), \tau_{2}=115.2 \mu \mathrm{s}(50.4 \%)\right]$. While the $\Phi_{\mathrm{PL}}$ largely benefits from the change in the host, the lifetimes of the prompt fluorescence remain largely unchanged while we observe a much shorter delayed fluorescence. Both prompt and delayed lifetimes of DICzTRZ in CzSi are shorter than those of ICzTRZ in the same host $\left(\tau_{\mathrm{p}} 9.5 \mathrm{~ns}, \tau_{\mathrm{d}}\right.$ of $147.3 \mu \mathrm{s}$, Supporting Information File 1, Figure S5f,g). The $\Delta E_{\mathrm{ST}}$ values in $\mathrm{CzSi}$ (Figure 5d) and PMMA (Supporting Information File 1, Figure $\mathrm{S} 5 \mathrm{~d}$ ), are $0.19 \mathrm{eV}$ and $0.03 \mathrm{eV}$, respectively. From a cross-comparison of the state energies (Table 1) we can see that the $\mathrm{T}_{1}$ state remains essentially the same regardless of the environment, this due to the LE nature of this excited state. The energy of the $S_{1}$ state varies with the environments (with energies of $2.94 \mathrm{eV}, 2.72 \mathrm{eV}$, and $2.75 \mathrm{eV}$ for toluene solution, $\mathrm{CzSi}$ film and PMMA film, respectively for ICzTRZ), characteristic of a CT type state, but the shape of the spectra in all media adopt a structured profile, typical for LE-type states, suggesting a state of mixed CT and LE character (Supporting Information File 1, Figures S4d and S5d). DICzTRZ and ICzTRZ possess comparable $\Delta E_{\mathrm{ST}}$ in $\mathrm{CzSi}$, at $0.19 \mathrm{eV}$ and $0.16 \mathrm{eV}$ respectively. Temperature-dependent time-resolved PL decays (Figure 5c) reveal the clear increase in the intensity of the delayed emission with higher temperature, a hallmark of TADF.

In our previous work, we investigated the orientation of ICzTRZ in a variety of guest-host systems prepared by co-evaporation [13,14]. In all of these systems ICzTRZ presented nearly-completely horizontal orientation with anisotropy values in the range 0.06 to 0.12 , depending on the host materials. However, in going from vacuum deposition, which was possible for the low-molecular weight emitter ICzTRZ, toward solution processing required for the much bigger DICzTRZ, one can expect significant changes of the orientation behaviour. It was shown, for example that phosphorescent iridium complexes like $\operatorname{Ir}(\mathrm{ppy})_{2}$ (acac) display horizontal orientation ( $a \cong 0.25$ ) after vacuum co-evaporation, while the orientation changed toward isotropic in spin-coated films with PMMA as the host [39]. Moreover, upon solution processing with a low- $T_{\mathrm{g}}$ host like $\mathrm{CBP}$, which is prone to crystallization, the obtained emitter orientation even turned vertical with the $a$ factor approaching 0.40 [39].

Thus, measurements of the anisotropy factor were accordingly carried out for both ICzTRZ and DICzTRZ. Polarization and angle dependent luminescence spectroscopy was used to measure $a$ for solution processed films of $20 \mathrm{wt} \%$ DICzTRZ in $\mathrm{CzSi}$. The data were then analyzed via optical simulation to yield and anisotropy factor of 0.53 , which disappointingly implies that the emitter presents a strongly vertical orientation (Figure 6); the corresponding measurement of $20 \mathrm{wt} \%$ 

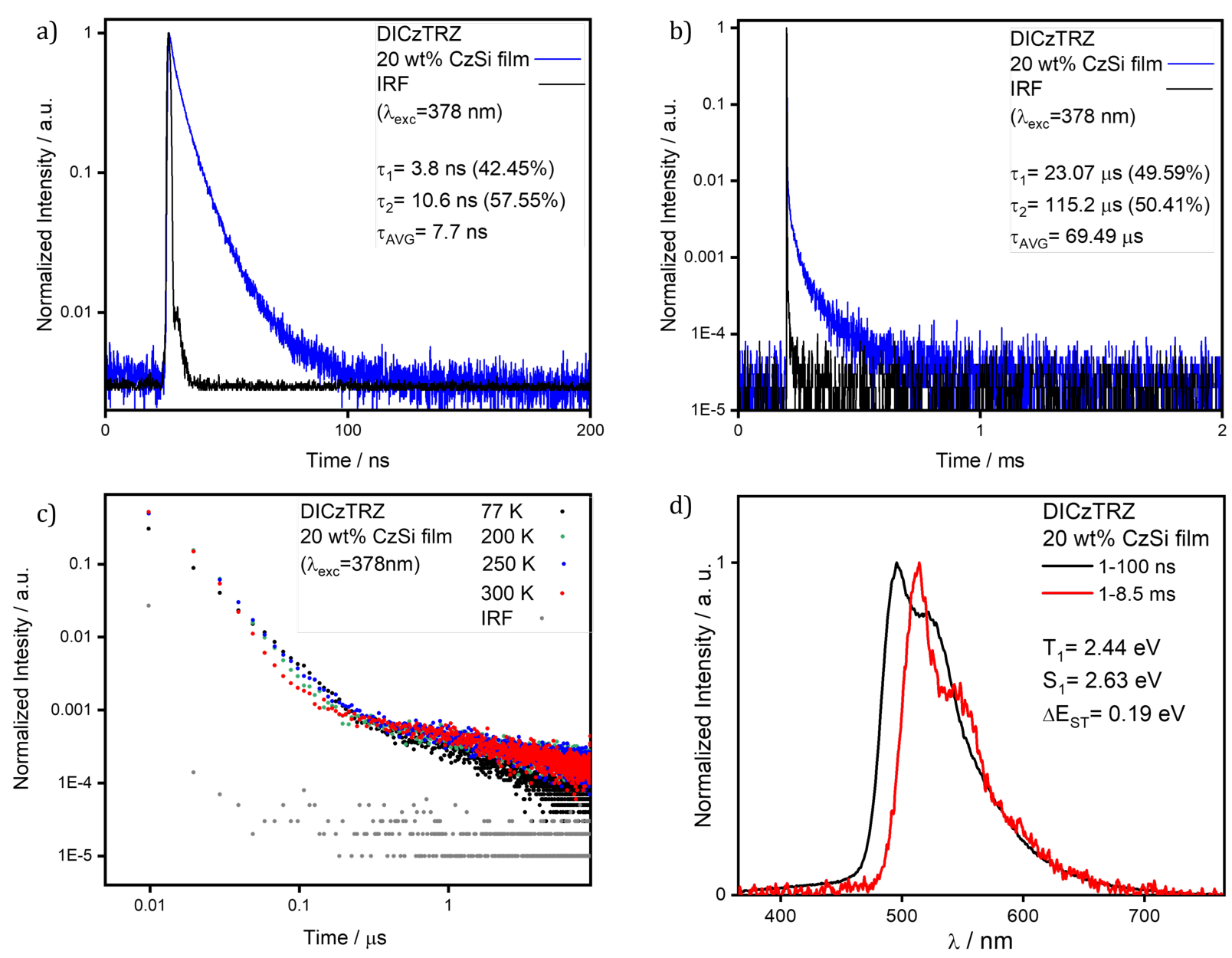

Figure 5: a) Prompt and b) delayed time-resolved decay in spin-coated $20 \mathrm{wt} \%$ CzSi film of DICzTRZ $\left(\lambda_{\text {exc }}=378 \mathrm{~nm}\right)$; c) delayed fluorescence decay data measured at different temperatures in spin-coated $20 \mathrm{wt} \% \mathrm{CzSi}$ film of DICzTRZ $\left(\lambda_{\text {exc }}=378 \mathrm{~nm}\right)$; d) prompt fluorescence and phosphorescence spectra at $77 \mathrm{~K}$ in drop-casted $20 \mathrm{wt} \% \mathrm{CzSi}$ film $\left(\lambda_{\mathrm{exc}}=343 \mathrm{~nm}\right.$, prompt and delayed fluorescence spectra were obtained in the 1-100 ns and $1-10 \mathrm{~ms}$ time range, respectively).

Table 1: Photophysical properties of ICzTRZ[15] and DICzTRZ.

\begin{tabular}{|c|c|c|c|c|c|c|c|}
\hline Material & Environment & $\lambda_{\mathrm{PL}} / \mathrm{nm}^{\mathrm{a}}$ & $\Phi_{\mathrm{PL}} \mathrm{N}_{2}(\text { air) })^{\mathrm{b}} / \%$ & $\mathrm{~T}_{\mathrm{p}}, \mathrm{T}_{\mathrm{d}}^{\mathrm{c}} / \mathrm{ns} ; \mu \mathrm{s}$ & $\mathrm{S}_{1}^{\mathrm{d}} / \mathrm{eV}$ & $\mathrm{T}_{1}^{\mathrm{e}} / \mathrm{eV}$ & $\Delta E_{\mathrm{ST}^{f}}^{\mathrm{f}} / \mathrm{eV}$ \\
\hline \multirow[t]{3}{*}{ ICzTRZg } & toluene $\left(10^{-5} \mathrm{M}\right)[15]$ & 462 & $72(56)^{h}$ & $9.0 ; 229.2$ & 2.94 & 2.62 & 0.32 \\
\hline & CzSi 20 wt \%i & 475 & $63(50)^{j}$ & $9.5 ; 147.3$ & 2.72 & 2.56 & 0.16 \\
\hline & PMMA 10 wt \%ं [15] & 470 & $31(28)^{j}$ & $115 ; 252.8$ & 2.75 & 2.64 & 0.11 \\
\hline \multirow[t]{3}{*}{ DICzTRZ $^{k}$} & toluene $\left(10^{-5} \mathrm{M}\right)$ & 477 & $60(44)^{h}$ & $8.9 ; 28.83$ & 2.80 & 2.59 & 0.21 \\
\hline & CzSi 20 wt \% ${ }^{i}$ & 488 & $57(42)^{j}$ & $7.7 ; 69.49$ & 2.63 & 2.44 & 0.19 \\
\hline & PMMA 10 wt \% ${ }^{i}$ & 514 & $29(22)^{j}$ & $8.6 ; 156.1$ & 2.61 & 2.58 & 0.03 \\
\hline
\end{tabular}

aMeasured at room temperature; ${ }^{b} \lambda_{\text {exc }}=340 \mathrm{~nm} ;{ }^{c} \mathrm{c}_{\mathrm{p}}$ (prompt lifetime) and $T_{\mathrm{d}}$ (delayed lifetime) were obtained from the transient PL decay of degassed solution/doped film, $\lambda_{\text {exc }}=378 \mathrm{~nm}$; ${ }^{d} S_{1}$ was obtained from the onset of the prompt emission measured at $77 \mathrm{~K}$; ${ }^{e} T_{1}$ was obtained from the onset of the phosphorescence spectrum measured at $77 \mathrm{~K} ;{ }^{f} \Delta E_{\mathrm{ST}}=\mathrm{S}_{1}-\mathrm{T}_{1}$. 9previous work [14]; hobtained via the optically dilute method [37] (see Supporting Information File 1), quinine sulfate $(0.5 \mathrm{M})$ in $\mathrm{H}_{2} \mathrm{SO}_{4}(\mathrm{aq})$ was used as the reference, $\Phi_{\mathrm{PL}}: 54.6 \%$ [38], $\lambda_{\mathrm{exc}}=360 \mathrm{~nm} ;{ }^{\mathrm{i}} \mathrm{spin}-\mathrm{coated}$ films; jobtained via integrating sphere; ${ }^{k}$ this work.

ICzTRZ in CzSi is shown in Supporting Information File 1, Clearly, both emitters exhibit unfavourable orientation of their Figure S6. It also shows vertical emitter orientation $(a=0.50$; TDMs when processed from solution. As stated above, this see fit in Supporting Information File 1, Figure S6). change of orientation in relation to the used processing condi- 


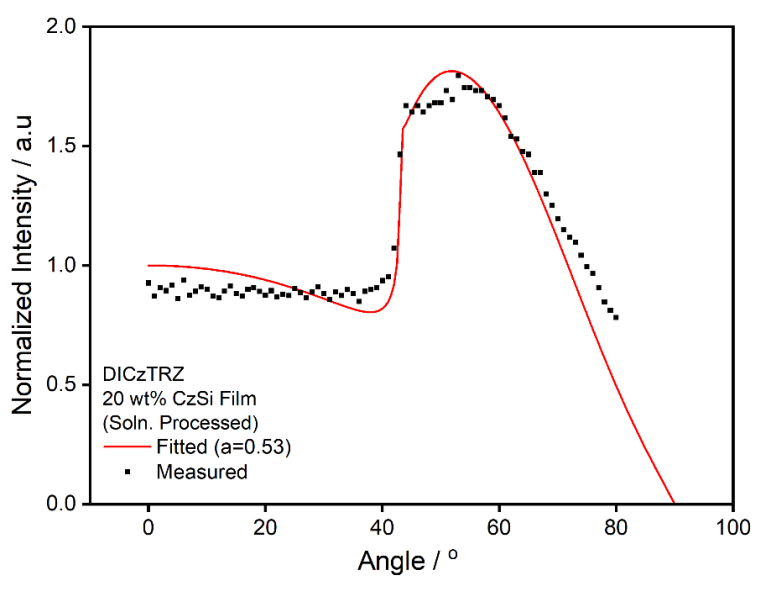

Figure 6: Angle-resolved photoluminescence measurement of a solution-processed film of $20 \mathrm{wt} \%$ DICzTRZ in CzSi. The red line shows a fit using the dipole emission model as described in detail in Supporting Information File 1, yielding an anisotropy factor, $a$, of 0.53 (data taken at $\lambda_{\mathrm{em}}=500 \mathrm{~nm}$ )

tions is not unexpected and confirms - once more - that an important driving force for non-isotropic emitter orientation upon vacuum deposition is the non-equilibrium situation at the surface of a growing film, as suggested by the Ediger group [40]. This is not the case for solution processing where molecules in the liquid film can almost freely rotate and adopt a more or less random orientation before the solvent evaporates and their orientation is fixed in the solid film. There may also be some effect of the host on the resulting orientation as well, which seems to be the case here for $\mathrm{CzSi}$ where we observe pronounced vertical orientation of both emitters. There is a difficulty in designing host molecules that lead emitters to orient horizontally without sacrificing other preferable properties of the host; for example, high triplet energy, good film-forming ability.

\section{OLED devices}

Finally, DICzTRZ and ICzTRZ-based OLEDs were fabricated using the following device structure: ITO (indium tin oxide) (50 nm)/PEDOT:PSS (poly(3,4-ethylenedioxythiophene) polystyrene sulfonate) $(35 \mathrm{~nm}) / \mathrm{PVK}$ (poly(9-vinylcarbazole)) $(10 \mathrm{~nm}) / X$ wt \% DICzTRZ or ICzTRZ: CzSi $(20 \mathrm{~nm}) / \mathrm{PPF}$ (2,8-bis(diphenylphosphoryl)dibenzo[b,d]furan) $(5 \mathrm{~nm}) / \mathrm{TPBi}$ (1,3,5-tris(1-phenyl-1H-benzo[d]imidazol-2-yl)benzene) $(50 \mathrm{~nm}) / \mathrm{Liq}$ (lithium quinolin-8-olate) $(1 \mathrm{~nm}) / \mathrm{Al}(80 \mathrm{~nm})$, where $X$ is 20 or 30 . The PVK layer is applied to facilitate hole injection from PEDOT:PSS to the emitting layer. Besides, PVK and PPF, possessing high $\mathrm{T}_{1}$ energies of $3.0 \mathrm{eV}$ [41] and $3.1 \mathrm{eV}$ [42], respectively, were inserted to confine the excitons in the emitting layer. PEDOT:PSS, PVK and the emitting layer were fabricated by spin-coating, and the other layers were vacuumdeposited. Device characteristics are shown in Figure 7 for DICzTRZ, Figure S7 (Supporting Information File 1) for ICzTRZ, and the device performance is summarized in Table 2. As shown in Table 2, 20 wt \% DICzTRZ-based OLEDs achieved $\mathrm{EQE}_{\max }$ of $8.4 \%$ and $\lambda_{\mathrm{EL}}$ of $494 \mathrm{~nm}$ with CIE coordinates $(x, y)$ of $(0.22,0.47)$ at $1 \mathrm{~mA} \mathrm{~cm}^{-2}$. The $20 \mathrm{wt} \%$ ICzTRZ-based OLEDs exhibited a slightly higher EQE max $_{\text {max }}$ of $11.6 \%$ and blue-shifted emission with $\lambda_{\mathrm{EL}}$ of $485 \mathrm{~nm}$. This result is consistent with that of the photophysical measurements for $20 \mathrm{wt} \%$ TADF emitter:CzSi films $\left(\Phi_{\mathrm{PL}}=57 \%\right.$ and $\lambda_{\mathrm{PL}}=488 \mathrm{~nm}$ for DICzTRZ, $\Phi_{\mathrm{PL}}=63 \%$ and $\lambda_{\mathrm{PL}}=475 \mathrm{~nm}$ for ICzTRZ, respectively).

We next simulated the device EQE (Supporting Information File 1, Figure S8). As shown in Figure 8, with the pre-determined parameters $\left(\Phi_{\mathrm{PL}}\right.$ and $\left.a\right)$ along with the optical constants of the different materials in the OLED stack, we predict the DICzTRZ device to show an $\mathrm{EQE}_{\max }$ of between $9-10 \%$, which aligns well with the measured $\mathrm{EQE}_{\max }$, whereas the corresponding solution-processed ICzTRZ OLED (see Supporting
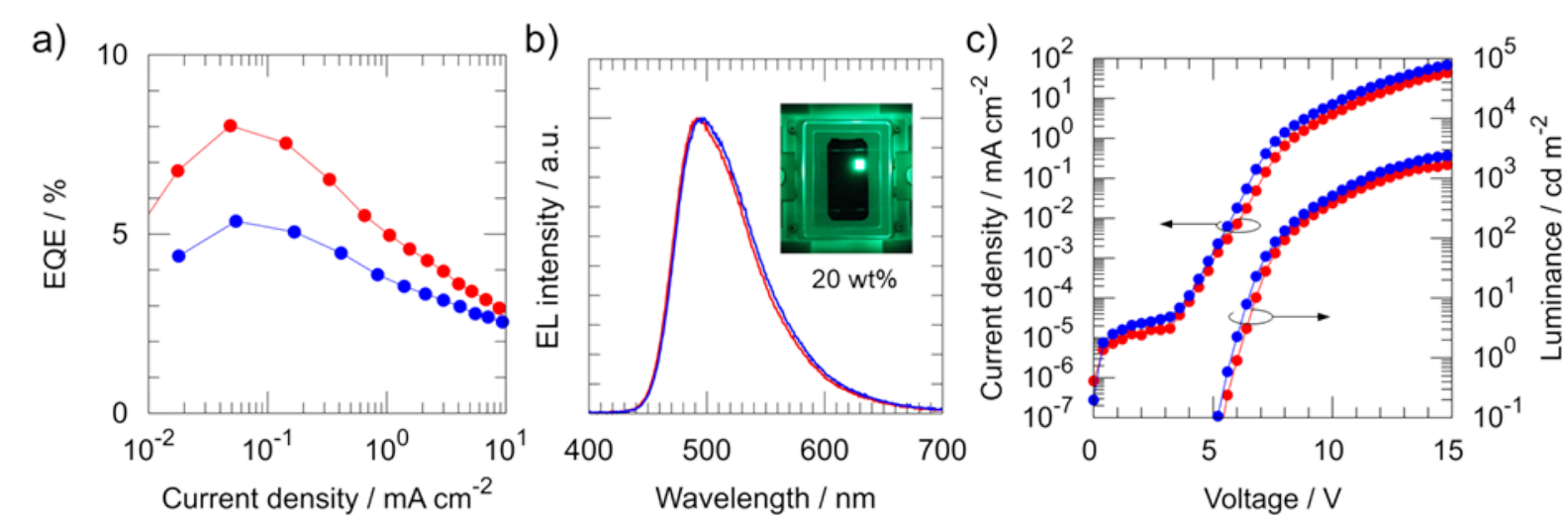

Figure 7: Device characteristics of 20 and 30 wt \% DICzTRZ-based OLEDs, which are represented by red and blue, respectively. a) EQE-current density, b) EL spectra and c) current density-voltage-luminance properties. 
Table 2: Device performances of $X$ wt \% ICzTRZ- and DICzTRZ-based OLEDs (where $X=20,30$ ).

\begin{tabular}{lllll} 
Emitter & Concentration $/ \%$ & $\mathrm{EQE}_{\max } / \%$ & $\lambda_{\mathrm{EL}} / \mathrm{nm}^{\mathrm{a}}$ & $\mathrm{CIE}(x, y)$ \\
\hline ICzTRZ & 20 & 11.6 & 485 & $(0.19,0.37)$ \\
& 30 & 6.6 & 485 & $(0.20,0.39)$ \\
\hline DICzTRZ & 20 & 8.4 & 494 & $(0.22,0.47)$ \\
& 30 & 5.4 & 498 & $(0.22,0.49)$ \\
\hline
\end{tabular}

aDetermined from EL spectrum at $1 \mathrm{~mA} \mathrm{~cm}^{-2}$.

Information File 1, Figure S7 for experimental data) is expected to show an $\mathrm{EQE}_{\max }$ of about $11 \%$, again in fairly good agreement with the measured results. The simulation also demonstrates that for ICzTRZ with the typically obtained alignment factor of about 0.1 in an evaporated device (and a slightly higher $\Phi_{\mathrm{PL}}$ of $70 \%$ as documented in ref. [14], a device EQE of about $22 \%$ can be expected as a result of the horizontal orientation of the emitter within an evaporated EML. Thus, vacuum deposition of this family of TADF emitters is clearly superior to solution processing.

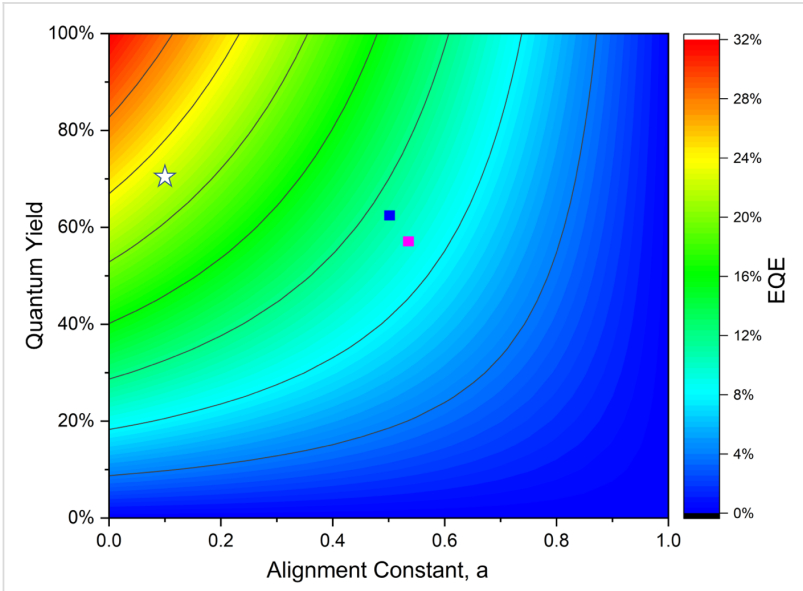

Figure 8: Device efficiency simulation of the fabricated OLEDs depicting the variation in EQE with varied PL quantum yield (vertical axis) and anisotropy factor (horizontal axis). The predicted EQEs are indicated with pink and blue rectangular marks for the DICzTRZ and ICzTRZ solution-processed OLEDs, respectively. The white star shows the predicted EQE for an evaporated ICzTRZ OLED with the orientation and PLQY taken from ref. [14]. All of the simulated EQEs agree fairly well with the experimental results.

\section{Conclusion}

Building upon our previously reported emitter, ICzTRZ, here we presented a dual emitter strategy consisting of two ICzTRZ moieties covalently linked together in the form of DICzTRZ. DFT calculations showed a much larger density of triplet states, which suggests that RISC will be faster in this compound compared to its parent. The twin design strategy leads to an enhancement in the molar extinction coefficient of the low-lying
CT states, accompanied by a red-shift in the emission. The 20 wt \% doped CzSi film of DICzTRZ emits in the blue at $488 \mathrm{~nm}$ and shows a photoluminescence quantum yield of $57.1 \%$. The $\Phi_{\mathrm{PL}}$ of DICzTRZ is slightly lower than that of ICzTRZ (63\% under $\mathrm{N}_{2}$ [14]), in line with its lower computed oscillator strength. DICzTRZ shows both prompt and delayed fluorescence, with a $\tau_{\mathrm{p}}$ that remains largely unchanged from that of ICzTRZ, while its $\tau_{\mathrm{d}}$ is significantly shorter. Unfortunately, the TDM of this material is not preferentially horizontally oriented in the solution-processed film, which is not unexpected in solution-processed films. The combination of its lower $\Phi_{\mathrm{PL}}$ and the vertical orientation of its TDM are the primary factors governing the relatively poorer device performance, with an $\mathrm{EQE}_{\max }$ of $8.4 \%$, compared to the vacuumdeposited OLED with ICzTRZ [14].

\section{Supporting Information}

The research data supporting this publication can be accessed at doi:10.17630/4a01d3e3-71bc-4ebb-9812-c4b838e13573.

\section{Supporting Information File 1}

Synthesis protocols, NMR spectra, supplementary photophysical measurements, computational data obtained from DFT and TD-DFT and electroluminescence data. [https://www.beilstein-journals.org/bjoc/content/ supplementary/1860-5397-17-197-S1.pdf]

\section{Supporting Information File 2}

$x y z$ Coordinates corresponding to the ground state optimized geometry of DICzTRZ.

[https://www.beilstein-journals.org/bjoc/content/ supplementary/1860-5397-17-197-S2.xyz]

\section{Funding}

We thank EU Horizon 2020 Grant Agreement No. 812872 (TADFlife) for funding this project. Further support was obtained by the Helmholtz Association Program at the Karlsruhe 
Institute of Technology (KIT). The German Research Foundation (formally Deutsche Forschungsgemeinschaft DFG) in the framework of SFB1176 Cooperative Research Centre "Molecular Structuring of Soft Matter" (CRC1176, A4, B3, C2, C6) and the cluster 3D Matter Made To Order all funded under Germany's Excellence Strategy -2082/1--390761711 are greatly acknowledged for financial contributions. We acknowledge support from the Engineering and Physical Sciences Research Council of the UK (grant EP/P010482/1), from the International Collaborative Research Program of Institute for Chemical Research, Kyoto University (grant \# 2020-37 and 2021-37), and from JSPS KAKENHI Grant Number JP20H05840 (Grant-inAid for Transformative Research Areas, "Dynamic Exciton"). $\mathrm{ZZ}$ acknowledges the financial support from the China Scholarship Council (CSC, 201606890009) for his PhD studies. EZ-C is a Royal Society Leverhulme Trust Senior Research fellow (SRF\R1\201089).

\section{ORCID ${ }^{\circledR}$ iDs}

Yoshimasa Wada - https://orcid.org/0000-0001-6139-8794 Prakhar Sahay - https://orcid.org/0000-0003-1692-5203 Wolfgang Brütting - https://orcid.org/0000-0001-9895-8281 Hironori Kaji - https://orcid.org/0000-0002-5111-3852 Stefan Bräse - https://orcid.org/0000-0003-4845-3191 Eli Zysman-Colman - https://orcid.org/0000-0001-7183-6022

\section{Preprint}

A non-peer-reviewed version of this article has been previously published as a preprint: https://doi.org/10.33774/chemrxiv-2021-sz2gb

\section{References}

1. Liu, Y.; Li, C.; Ren, Z.; Yan, S.; Bryce, M. R. Nat. Rev. Mater. 2018, 3 , 18020. doi:10.1038/natrevmats.2018.20

2. Wong, M. Y.; Zysman-Colman, E. Adv. Mater. (Weinheim, Ger.) 2017, 29, 1605444. doi:10.1002/adma.201605444

3. Baldo, M. A.; O'Brien, D. F.; You, Y.; Shoustikov, A.; Sibley, S.; Thompson, M. E.; Forrest, S. R. Nature 1998, 395, 151-154. doi:10.1038/25954

4. Milián-Medina, B.; Gierschner, J. Org. Electron. 2012, 13, 985-991. doi:10.1016/j.orgel.2012.02.010

5. Cui, L.-S.; Nomura, H.; Geng, Y.; Kim, J. U.; Nakanotani, H.; Adachi, C. Angew. Chem., Int. Ed. 2017, 56, 1571-1575. doi:10.1002/anie.201609459

6. Rajamalli, P.; Chen, D.; Li, W.; Samuel, I. D. W.; Cordes, D. B.; Slawin, A. M. Z.; Zysman-Colman, E. J. Mater. Chem. C 2019, 7, 6664-6671. doi:10.1039/c9tc01498e

7. dos Santos, P. L.; Chen, D.; Rajamalli, P.; Matulaitis, T.; Cordes, D. B.; Slawin, A. M. Z.; Jacquemin, D.; Zysman-Colman, E.; Samuel, I. D. W. ACS Appl. Mater. Interfaces 2019, 11, 45171-45179. doi:10.1021/acsami.9b16952

8. Dias, F. B.; Penfold, T. J.; Monkman, A. P. Methods Appl. Fluoresc. 2017, 5, 012001. doi:10.1088/2050-6120/aa537e

9. El-Sayed, M. A. Acc. Chem. Res. 1968, 1, 8-16. doi:10.1021/ar50001a002
10. Schmidt, T. D.; Lampe, T.; Sylvinson M. R., D.; Djurovich, P. I.; Thompson, M. E.; Brütting, W. Phys. Rev. Appl. 2017, 8, 037001. doi:10.1103/physrevapplied.8.037001

11. Xiang, S.; Lv, X.; Sun, S.; Zhang, Q.; Huang, Z.; Guo, R.; Gu, H.; Liu, S.; Wang, L. J. Mater. Chem. C 2018, 6, 5812-5820. doi:10.1039/c8tc01419a

12. Maeng, J. H.; Ahn, D. H.; Lee, H.; Jung, Y. H.; Karthik, D.; Lee, J. Y.; Kwon, J. H. Dyes Pigm. 2020, 180, 108485. doi:10.1016/j.dyepig.2020.108485

13. Naqvi, B. A.; Schmid, M.; Crovini, E.; Sahay, P.; Naujoks, T.; Rodella, F.; Zhang, Z.; Strohriegl, P.; Bräse, S.; Zysman-Colman, E.; Brütting, W. Front. Chem. (Lausanne, Switz.) 2020, 8, 750. doi:10.3389/fchem.2020.00750

14. Zhang, Z.; Crovini, E.; dos Santos, P. L.; Naqvi, B. A.; Cordes, D. B.; Slawin, A. M. Z.; Sahay, P.; Brütting, W.; Samuel, I. D. W.; Bräse, S.; Zysman-Colman, E. Adv. Opt. Mater. 2020, 8, 2001354. doi:10.1002/adom.202001354

15. Park, H.-J.; Han, S. H.; Lee, J. Y. J. Mater. Chem. C 2017, 5, 12143-12150. doi:10.1039/c7tc03133e

16. Cho, Y. J.; Jeon, S. K.; Chin, B. D.; Yu, E.; Lee, J. Y. Angew. Chem., Int. Ed. 2015, 54, 5201-5204. doi:10.1002/anie.201412107

17. Kim, M.; Jeon, S. K.; Hwang, S.-H.; Lee, S.-S.; Yu, E.; Lee, J. Y. Chem. Commun. 2016, 52, 339-342. doi:10.1039/c5cc07999c

18. Wei, D.; Ni, F.; Wu, Z.; Zhu, Z.; Zou, Y.; Zheng, K.; Chen, Z.; Ma, D.; Yang, C. J. Mater. Chem. C 2018, 6, 11615-11621. doi:10.1039/c8tc02849d

19. Chen, D.; Kusakabe, Y.; Ren, Y.; Sun, D.; Rajamalli, P.; Wada, Y.; Suzuki, K.; Kaji, H.; Zysman-Colman, E. J. Org. Chem. 2021, 86, 11531-11544. doi:10.1021/acs.joc.1c01101

20. Byeon, S. Y.; Kim, J.; Lee, D. R.; Han, S. H.; Forrest, S. R.; Lee, J. Y. Adv. Opt. Mater. 2018, 6, 1701340. doi:10.1002/adom.201701340

21. Sharma, N.; Spuling, E.; Mattern, C. M.; Li, W.; Fuhr, O.; Tsuchiya, Y.; Adachi, C.; Bräse, S.; Samuel, I. D. W.; Zysman-Colman, E. Chem. Sci. 2019, 10, 6689-6696. doi:10.1039/c9sc01821b

22. Kim, M.; Jeon, S. K.; Hwang, S.-H.; Lee, S.-S.; Yu, E.; Lee, J. Y. J. Phys. Chem. C 2016, 120, 2485-2493. doi:10.1021/acs.jpcc.5b09114

23. Senes, A.; Meskers, S. C. J.; Dijkstra, W. M.; van Franeker, J. J.; Altazin, S.; Wilson, J. S.; Janssen, R. A. J. J. Mater. Chem. C 2016, 4, 6302-6308. doi:10.1039/c5tc03481g

24. Senes, A.; Meskers, S. C. J.; Greiner, H.; Suzuki, K.; Kaji, H.; Adachi, C.; Wilson, J. S.; Janssen, R. A. J. J. Mater. Chem. C 2017, 5, 6555-6562. doi:10.1039/c7tc01568b

25. Gao, Y.; Hlil, A.; Wang, J.; Chen, K.; Hay, A. S. Macromolecules 2007, 40, 4744-4746. doi:10.1021/ma0702750

26. Zhang, Q.; Zhuang, H.; He, J.; Xia, S.; Li, H.; Li, N.; Xu, Q.; Lu, J. J. Mater. Chem. C 2015, 3, 6778-6785. doi:10.1039/c5tc00839e

27. Grimme, S. Chem. Phys. Lett. 1996, 259, 128-137. doi:10.1016/0009-2614(96)00722-1

28. Santos, P. L.; Ward, J. S.; Data, P.; Batsanov, A. S.; Bryce, M. R.; Dias, F. B.; Monkman, A. P. J. Mater. Chem. C 2016, 4, 3815-3824. doi:10.1039/c5tc03849a

29. Hosokai, T.; Matsuzaki, H.; Nakanotani, H.; Tokumaru, K.; Tsutsui, T.; Furube, A.; Nasu, K.; Nomura, H.; Yahiro, M.; Adachi, C. Sci. Adv. 2017, 3, e1603282. doi:10.1126/sciadv.1603282

30. Noda, H.; Nakanotani, H.; Adachi, C. Sci. Adv. 2018, 4, eaao6910. doi:10.1126/sciadv.aao6910

31. Samanta, P. K.; Kim, D.; Coropceanu, V.; Brédas, J.-L. J. Am. Chem. Soc. 2017, 139, 4042-4051. doi:10.1021/jacs.6b12124 
32. Wada, Y.; Nakagawa, H.; Matsumoto, S.; Wakisaka, Y.; Kaji, H. ChemRxiv 2019, (ii), 1-21. doi:10.26434/chemrxiv.9745289.v1

33. Wada, Y.; Nakagawa, H.; Matsumoto, S.; Wakisaka, Y.; Kaji, H. Nat. Photonics 2020, 14, 643-649. doi:10.1038/s41566-020-0667-0

34. Cha, J.-R.; Lee, C. W.; Lee, J. Y.; Gong, M.-S. Dyes Pigm. 2016, 134, 562-568. doi:10.1016/j.dyepig.2016.08.023

35. Cho, Y. J.; Jeon, S. K.; Lee, S.-S.; Yu, E.; Lee, J. Y. Chem. Mater. 2016, 28, 5400-5405. doi:10.1021/acs.chemmater.6b01484

36. Wang, X.; Yan, Q.; Chu, P.; Luo, Y.; Zhang, Z.; Wu, S.; Wang, L.; Zhang, Q. J. Lumin. 2011, 131, 1719-1723. doi:10.1016/j.jlumin.2011.03.061

37. Crosby, G. A.; Demas, J. N. J. Phys. Chem. 1971, 75, 991-1024. doi:10.1021/j100678a001

38. Melhuish, W. H. J. Phys. Chem. 1961, 65, 229-235. doi:10.1021/j100820a009

39. Lampe, T.; Schmidt, T. D.; Jurow, M. J.; Djurovich, P. I.; Thompson, M. E.; Brütting, W. Chem. Mater. 2016, 28, 712-715. doi:10.1021/acs.chemmater.5b04607

40. Ediger, M. D.; de Pablo, J.; Yu, L. Acc. Chem. Res. 2019, 52, 407-414. doi:10.1021/acs.accounts.8b00513

41. Kumar, M.; Pereira, L. ACS Omega 2020, 5, 2196-2204. doi:10.1021/acsomega.9b03253

42. Vecchi, P. A.; Padmaperuma, A. B.; Qiao, H.; Sapochak, L. S.; Burrows, P. E. Org. Lett. 2006, 8, 4211-4214. doi:10.1021/ol0614121

\section{License and Terms}

This is an open access article licensed under the terms of the Beilstein-Institut Open Access License Agreement (https://www.beilstein-journals.org/bjoc/terms), which is identical to the Creative Commons Attribution 4.0 International License (https://creativecommons.org/licenses/by/4.0). The reuse of material under this license requires that the author(s), source and license are credited. Third-party material in this article could be subject to other licenses (typically indicated in the credit line), and in this case, users are required to obtain permission from the license holder to reuse the material.

The definitive version of this article is the electronic one which can be found at: https://doi.org/10.3762/bjoc.17.197 